\title{
Mathematical Computation of Couple Stress Fluid Flow Through Stenosed Artery With Suspension of Nanoparticles
}

\author{
K. Maruthi Prasad, T. Sudha
}

\begin{abstract}
This work consists the effect of an overlapping constriction of couple stress fluid with nanoparticles. The equations are derived analytically by using HPM technique. Effects of relevant parameters on flow resistance and shear stress at wall of the fluid are investigated. It is observed that flow resistance increases stenosis height and Brownian motion parameter. Flow resistance reduces with local nanoparticle and temperature Grashof numbers, couple stress fluid parameters and thermophoresis parameter.
\end{abstract}

Keywords: Couple stress fluid, Overlapping stenosis, Nanoparticles, Resistance to the flow.

\section{INTRODUCTION}

The circulatory system or cardiovascular system supplies blood, oxygen and nutrients to throughout the body. Cardiovascular system is the hardest working organ in the human body. In the present world majority of deaths are related to cardiovascular diseases. In earlier days cardiac diseases affects the aged group. But in recent days, cardiac diseases are attacking in young people also. If this continues the future looks more dangerous. The main reason for this situation is less exercise, stress, eating junk food, pollution, smoking and drinking alcohol. Stenosis is one of the major cardiac diseases and it is define as narrowing of an artery with fatty substances. Stenosis blocks the blood vessel partially or fully and it leads to heart attacks, brain strokes and hypertension etc.

Stenosis was first investigated by Young [1]. Later Young and different researchers have analyzed the blood flow problems in narrowed vessels by taking blood as Newtonian or non- Newtonian fluids under different situations (Misra and Chakravarthy [2], Srivastava and Mishra [3], Maruthi Prasad et al., [4]).

In the recent days, study of couple stress fluid has gained much attention. It was initiated by Stokes [5] have distinct properties, like occurrence of body couples, couple stresses and non-symmetric stress tensor, mathematical simplicity compared with other models.

Revised Manuscript Received on December 15, 2019.

K. Maruthi Prasad, Department of Mathematics, School of Technology, GITAM University, Hyderabad 502 329, TELANGANA, INDIA. Email: maruthiprasad.karanamu@ gitam.edu

T. Sudha, Department of Mathematics, Malla Reddy College of Engineering for Women, Hyderabad 500 014, TELANGANA, INDIA.

*Email: sudhathulluri@gmail.com
Nanofluid is the next exciting frontier in technology. Nanofluid have distinct features such as, enhanced thermal diffusivity, thermal conductivity, convective heat transfer coefficients and viscosity. Mekheimer et al., [6] studied about MHD blood flows in an artery with overlapping stenosis with metallic nanoparticles effects. Maruthi Prasad and Sudha [7] presented the mathematical model of couple stress fluid flows through the multiple constricted tubes with suspension of nanoparticles.

This paper deals with overlapping constriction effects of a couple-stress fluid with nanoparticles. The analysis is done analytically using mild stenosis approximation. Expressions for flow resistance and wall shear stress are calculated.

\section{PROBLEM FORMULATION}

In this study, Considered the couple stress fluid flow with nanoparticles in an axisymmetric tube with overlapping constriction is depicted in fig. 1 . The geometry of abnormality can be considered from (Srivastava and Mishra, [3]) 


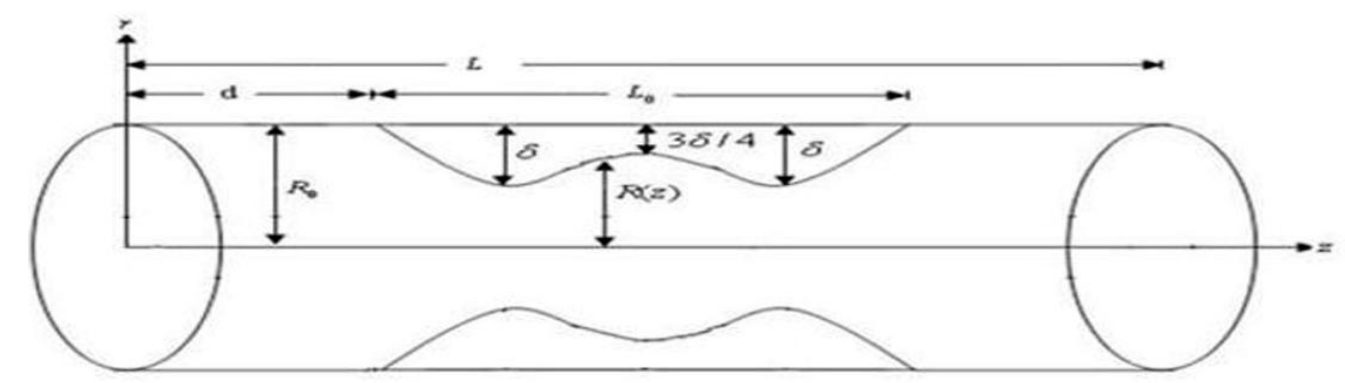

Fig. 1. Graphical representation of the tube with constriction

$h=\frac{R(z)}{R_{0}}=\left\{\begin{array}{rr}1-\frac{3 \delta}{2 R_{0} L_{0}^{4}}\left[11(z-d) L_{0}^{3}-47(z-d)^{2} L_{0}^{2}+72(z-d)^{3} L_{0}-36(z-d)^{4}\right], \\ 1, & \text { for } d \leq z \leq d+L_{0}, \\ \text { otherwise } .\end{array}\right.$

Where $R(z)$ denotes tube radius with constriction, $R_{0}(z)$ gives tube radius without constriction, $L_{0}$ denotes the stenosis length and $d$ denotes its location, $\delta$ denotes highest height of the constriction situated at the two positions is taken as $z=d+\frac{L_{0}}{6}, z=d+\frac{5 L_{0}}{6}$ respectively. The critical height is $\frac{3 \delta}{4}$ at $z=d+L_{0} / 2$, from the origin.

Using non-dimensional quantities

$\bar{d}=\frac{d}{L}, \bar{z}=\frac{z}{L}, \quad \bar{L}=\frac{L}{L_{0}}, \quad \bar{u}=\frac{u}{U}, \bar{R}(z)=\frac{R(z)}{R_{0}}, \quad \bar{\delta}=\frac{\delta}{R_{0}}$,

$\bar{u}=\frac{u}{U}, \bar{P}=\frac{P}{\mu U L / R_{0}^{2}}, \bar{q}=\frac{q}{\pi R_{0}^{2} U}, \quad R_{e}=\frac{2 \rho c_{1} R_{0}}{\mu}, \bar{h}=\frac{h}{h_{0}}$,

$N_{b}=\frac{(\rho C)_{P} D_{\bar{B}} \bar{C}_{0}}{(\rho C)_{f}}, \quad N_{t}=\frac{(\rho C)_{P} D_{T} \bar{T}_{0}}{(\rho C)_{f} \beta}, \quad G_{r}=\frac{g \beta \bar{T}_{0} R_{0}^{3}}{\gamma^{2}}, \quad B_{r}=\frac{g \beta \bar{C}_{0} R_{0}^{3}}{\gamma^{2}}$.

Incompressible couple stress fluid equations with nanoparticles under hypothesis of mild stenosis condition are taken from Maruthi Prasad and Sudha [7]

$$
\begin{gathered}
\frac{1}{r} \frac{\partial}{\partial r}\left(r \frac{\partial}{\partial r}\left(1-\frac{1}{\bar{\alpha}^{2}} \nabla^{2}\right) u\right)=\frac{d p}{d z}+G_{r} \theta_{t}+B_{r} \sigma \\
0=\frac{1}{r} \frac{\partial}{\partial r}\left(r \frac{\partial \theta_{t}}{\partial r}\right)+N_{b} \frac{\partial \sigma}{\partial r} \frac{\partial \theta_{t}}{\partial r}+N_{t}\left(\frac{\partial \theta_{t}}{\partial r}\right)^{2} \\
0=\frac{1}{r} \frac{\partial}{\partial r}\left(r \frac{\partial \sigma}{\partial r}\right)+\frac{N_{t}}{N_{b}}\left(\frac{1}{r} \frac{\partial}{\partial r}\left(r \frac{\partial \theta_{t}}{\partial r}\right)\right)
\end{gathered}
$$

$\bar{\alpha}^{2}=\frac{\mu}{\eta} a^{2}$ (Couple stress parameter), $u$ represents averaged velocity over part of the tube with $R_{0}$ (radius). $\theta_{t} \& \sigma$ denotes temperature profile and nanoparticle phenomena. Local temperature Grashof number, Brownian motion parameter, local nanoparticle Grashof number and thermophoresis parameter are represented by $G_{r}, N_{b}, B_{r} \& N_{t}$.

The non-dimensional boundary equations are

$$
\begin{gathered}
\frac{\partial u}{\partial r}=0, \frac{\partial \theta_{t}}{\partial r}=0, \frac{\partial \sigma}{\partial r}=0 \text { at } r=0 \\
u=0, \theta_{t}=0, \sigma=0 \text { at } r=h(z), \\
\frac{\partial^{2} u}{\partial r^{2}}-\frac{\eta}{r} \frac{\partial u}{\partial r}=0 \text { at } r=h(z), \\
\frac{\partial^{2} u}{\partial r^{2}}-\frac{\eta}{r} \frac{\partial u}{\partial r} \text { is finite at } r=0 .
\end{gathered}
$$

\section{SOLUTION}

The solution of the coupled Equations (3) and (4) are derived by using HPM (Homotopy perturbation method) as

$$
H\left(q_{t}, \theta_{t}\right)=\left(1-q_{t}\right)\left[L\left(\theta_{t}\right)-L\left(\theta_{t_{10}}\right)\right]+q_{t}\left[L\left(\theta_{t}\right)+N_{b} \frac{\partial \sigma}{\partial r} \frac{\partial \theta_{t}}{\partial r}+\quad N_{t}\left(\frac{\partial \theta_{t}}{\partial r}\right)^{2}\right]
$$

(9)

$H\left(q_{t}, \sigma\right)=\left(1-q_{t}\right)\left[L(\sigma)-L\left(\sigma_{10}\right)\right]+q_{t}\left[L(\sigma)+\frac{N_{t}}{N_{b}}\left(\frac{1}{r} \frac{\partial}{\partial r}\left(r \frac{\partial \theta_{t}}{\partial r}\right)\right)\right]$. 
. For ease, $L=\frac{1}{r} \frac{\partial}{\partial r}\left(r \frac{\partial}{\partial r}\right)$ is considered as linear operator. The initial guesses $\theta_{t_{10}}$ and $\sigma_{10}$ are taken as

$$
\theta_{10}(r, z)=\left(\frac{r^{2}-h^{2}}{4}\right), \quad \sigma_{10}(r, z)=-\left(\frac{r^{2}-h^{2}}{4}\right)
$$

Adopt the same process as done by Maruthi Prasad and Sudha [7], the equations for nanoparticle and temperature profiles for $q_{t}=1$

$$
\sigma(r, z)=-\left(\frac{r^{2}-h^{2}}{4}\right) \frac{N_{t}}{N_{b}}, \theta_{t}(r, z)=\left(\frac{r^{4}-h^{4}}{64}\right)\left(N_{b}-N_{t}\right)
$$

Substituting the Equation (12), in the Equation (2) and by using boundary conditions, the expression for the velocity is $u(r, z)=A I_{0}(\bar{\alpha} r)+\frac{r^{6} T}{2304}+\frac{r^{4}}{64}\left(\frac{T}{\bar{\alpha}^{2}}-S\right)+\frac{r^{2}}{4}\left(\frac{T}{\bar{\alpha}^{4}}-\frac{T h^{4}}{64}+\frac{h^{2} S}{4}-\frac{S}{\bar{\alpha}^{2}}\right)+T\left(\frac{1}{\bar{\alpha}^{6}}-\frac{h^{4}}{64 \bar{\alpha}^{2}}\right)+S\left(\frac{h^{2}}{4 \bar{\alpha}^{2}}-\frac{1}{\bar{\alpha}^{4}}\right)+\left(\frac{d p}{d z}\right)\left(\frac{r^{2}}{4}+\frac{1}{\bar{\alpha}^{2}}\right)-$ $d \bar{\alpha}^{2}$.

Where $T=G_{r}\left(N_{b}-N_{t}\right), S=B_{r}\left(\frac{N_{t}}{N_{b}}\right)$

$$
\begin{gathered}
A=\frac{\left[\frac{h(\eta-1)}{2}\left(\frac{d p}{d z}+\frac{T}{\bar{\alpha}^{4}}-\frac{T h^{4}}{64}+\frac{h^{2} S}{4}-\frac{S}{\bar{\alpha}^{2}}\right)+(\eta-5) \frac{T h^{5}}{384}+\frac{h^{3}}{16}(\eta-3)\left(\frac{T}{\bar{\alpha}^{2}}-S\right)\right]}{h \bar{\alpha}^{2} I_{0}(\bar{\alpha} h)-\bar{\alpha} I_{1}(\bar{\alpha} h)-\eta \bar{\alpha} I_{1}(\bar{\alpha} h)} \\
d=\frac{1}{\bar{\alpha}^{2}}\left[\begin{array}{c}
\left.A I_{0}(\bar{\alpha} h)+\frac{h^{6} T}{2304}+\frac{h^{4}}{64}\left(\frac{T}{\bar{\alpha}^{2}}-S\right)+\frac{h^{2}}{4}\left(\frac{T}{\bar{\alpha}^{4}}-\frac{T h^{4}}{64}+\frac{h^{2} S}{4}-\frac{S}{\bar{\alpha}^{2}}\right)+\right] \\
T\left(\frac{1}{\bar{\alpha}^{6}}-\frac{h^{4}}{64 \bar{\alpha}^{2}}\right)+S\left(\frac{h^{2}}{4 \bar{\alpha}^{2}}-\frac{1}{\bar{\alpha}^{4}}\right)+\left(\frac{d p}{d z}\right)\left(\frac{h^{2}}{4}+\frac{1}{\bar{\alpha}^{2}}\right)
\end{array}\right]
\end{gathered}
$$

The dimension less flux $q$ is $q=\int_{0}^{h} 2 r u d r$.

By substituting the Equation (13) in Equation (14), then the flux is

$q=A\left(\frac{2 h}{\bar{\alpha}} I_{1}(\bar{\alpha} h)-h^{2} I_{0}(\bar{\alpha} h)\right)-\frac{h^{4}}{8}\left(\frac{d p}{d z}\right)-\frac{T h^{8}}{3072}-\frac{h^{6}}{96}\left(\frac{T}{\bar{\alpha}^{2}}-S\right)-\quad \frac{h^{4}}{8}\left(\frac{T}{\bar{\alpha}^{4}}-\frac{T h^{4}}{64}+\frac{h^{2} S}{4}-\frac{S}{\bar{\alpha}^{2}}\right)$

From Equation (15), $\frac{d P}{d z}$ can be given as

$\frac{d p}{d z}=\frac{8 q}{M h^{4}}+\frac{T}{M}\left[\frac{h^{4}}{384}-\frac{h D(\eta-5)}{48 K}\right]-\left[\frac{T}{\bar{\alpha}^{4}}-\frac{T h^{4}}{64}+\frac{h^{2} S}{4}-\frac{S}{\bar{\alpha}^{2}}\right]+\quad \frac{1}{M}\left[\left(\frac{T}{\bar{\alpha}^{2}}-S\right)\left(\frac{h^{2}}{12}-\frac{D(\eta-3}{2 K h}\right)\right]$

(16)

Where $K=h \bar{\alpha}^{2} I_{0}(\bar{\alpha} h)-\bar{\alpha} I_{1}(\bar{\alpha} h)-\eta \bar{\alpha} I_{1}(\bar{\alpha} h)$

$$
D=\frac{2 h}{\bar{\alpha}} I_{1}(\bar{\alpha} h)-h^{2} I_{0}(\bar{\alpha} h), \quad M=\frac{4 D(\eta-1)}{K h^{3}}-1
$$

The pressure drop over one wave length $p(0)-p(\lambda)$ is

$\Delta p=-\int_{0}^{1} \frac{d p}{d z}$

The flow resistance $\lambda=\frac{\Delta p}{q}$

Pressure drop without abnormality $(h=1)$ is defined as

$\Delta p_{n}=\left[-\int_{0}^{1} \frac{d p}{d z} d z\right]_{h=1}$

The flow resistance in the regular artery is $\lambda_{n}=\frac{\Delta p_{n}}{q}$

The normalized flow resistance $\bar{\lambda}=\frac{\lambda}{\lambda_{n}}$

And the shear stress at wall $\tau_{h}=-\frac{h}{2} \frac{d p}{d z}$

\section{RESULTS AND ANALYSIS}

Figures (2) - (6) presents the effects of flow variables for different parameters by taking parameter values as $L_{0}=0.2,0.3,0.4, d=0.2, q=0.1, L=1, \delta=$ $0,0.02,0.04,0.06,0.08,0.1, B_{r}=0.3, G_{r}=0.2, N_{b}=$
$0.3, N_{t}=0.8$ (Maruthi Prasad et al., [4] and Maruthi Prasad and Sudha [7]).

Figures (2) - (4) explain the effects of $\bar{\lambda}$ (flow resistance) with $\delta$ (stenosis height) for relevant pa-

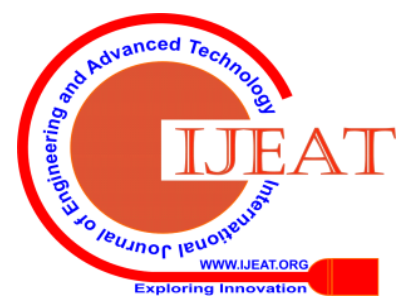


rameters. It is identified that flow resistance enhances with the stenosis height $(\delta)$ and $N_{b}$ (Brownian motion parameter). It reduces with couple stress fluid parameters $(\bar{\alpha}, \eta)$, local temperature and nanoparticle numbers $\left(G_{r} \& B_{r}\right)$ and $N_{t}$ (thermophoresis parameter).

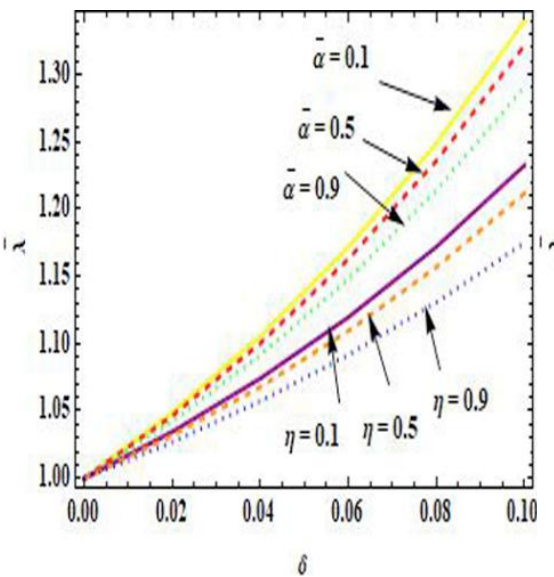

Fig. 2. Representation of $\delta, \bar{\alpha} \& \eta$ on $\bar{\lambda}$

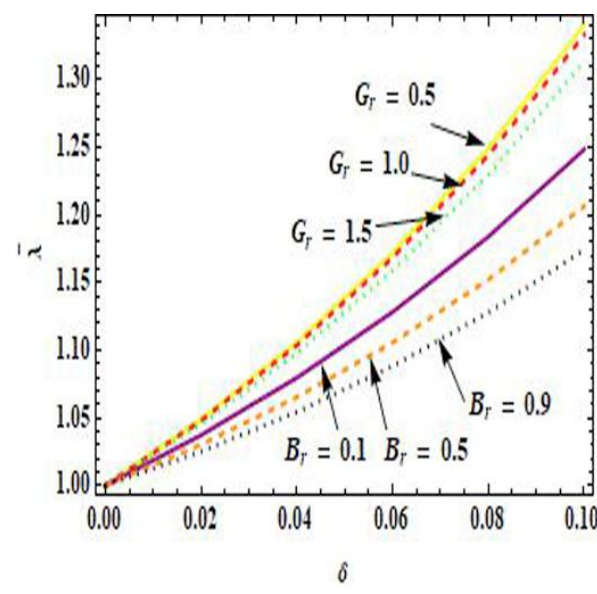

Fig. .4. Representation of $\delta, G_{r} \& B_{r}$ on $\bar{\lambda}$

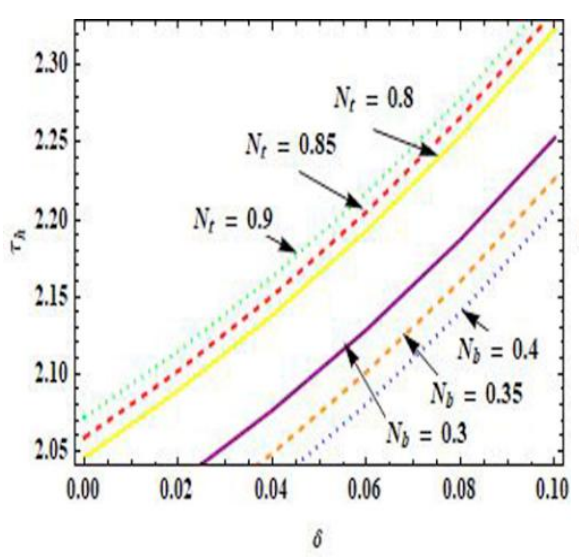

Fig. 6. Representation $\delta, N_{t} \& N_{b}$ on $\tau_{h}$
Figures (5) - (7) shows that $\tau_{h}$ (wall shear stress) enhances with thermophoresis parameter $\left(N_{t}\right)$, stenosis height $(\delta)$, local temperature and nanoparticle numbers $\left(G_{r} \& B_{r}\right)$. But reduces with $\bar{\alpha}, \eta$ (couple stress fluid parameters) and $N_{b}$ (Brownian motion parameter).

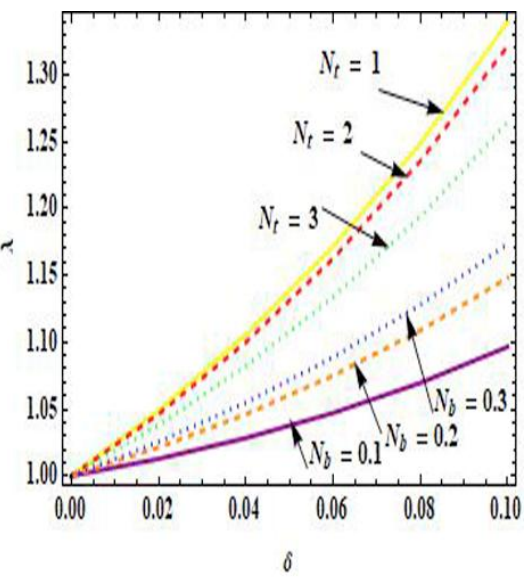

Fig. 3. Representation of $\delta, N_{t} \& N_{b}$ on $\bar{\lambda}$

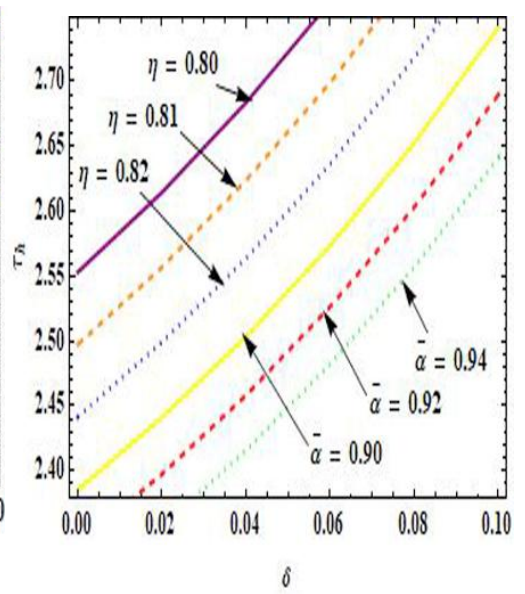

Fig. 5. Representation $\delta, \bar{\alpha} \& \eta$ on $\tau_{h}$

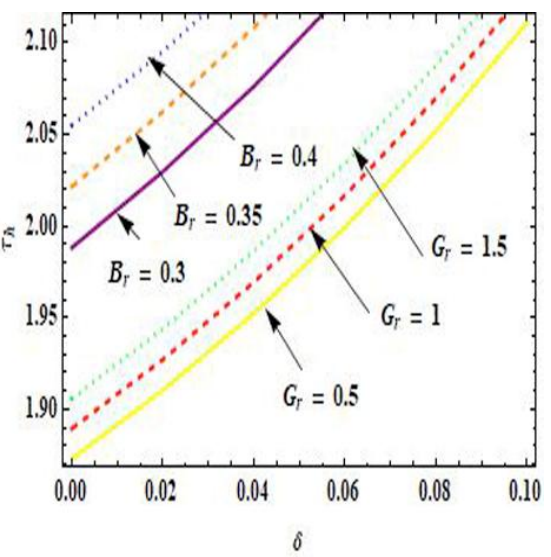

Fig. 7. Representation $\delta, G_{r} \& B_{r}$ on $\tau_{h}$ 


\section{CONCLUSION}

A theoretical model for an incompressible and steady flows of couple stress fluid with nanoparticles through a tube consisting overlapping constriction has been investigated. It is observed that flow resistance enhances with Brownian motion parameter and stenosis height. It reduces with couple stress fluid parameters, local nanoparticle and temperature Grashof numbers \& thermophoresis parameter.

\section{REFERENCES}

1. Young, D.F.: Effect of a time-dependent stenosis on flow through a tube. Trans. ASME J. Engng Ind. 90, 248-254 (1968).https://doi.org/10.1115/1.3604621

2. Misra, J.C., Chakravarthy, S.: Flow in arteries in the presence of Stenosis. Journal of Biomechanics, 19, 907918 (1986). $\quad$ https://doi.org/10.1016/00219290(86)90186-7

3. Srivastava, V. P., Shailesh Mishra.: Non-newtonian arterial blood flow through an overlapping stenosis. Applications of Applied Mathematics, 5, 225-238 (2010).

4. Maruthi Prasad, K., Bhuvana Vijaya, R., Umadevi, C.: A mathematical model of Herschel- Bulkley fluid through an overlapping stenosis. IOSR Journal of Mathematics.10,41-46, (2014). https://doi.org/10.9790/572810224146

5. Stokes, V. K.: Couple Stresses in Fluids, AIP Physics of $\begin{array}{llll}\text { Fluids. } & 9, & 1709 & \end{array}$ https://doi.org/10.1063/1.1761925

6. Mekheimer, K. S., Mohamed, M. S., Elnaqeeb, T.: Metallic nanoparticles influence on blood flow through a stenotic artery. International Journal of Pure and Applied Mathematics, 107,201-220

(2016). https://doi.org/10.12732/ijpam.v107i1.16

7. Maruthi Prasad, K., Sudha T.: Mathematical analysis of non-newtonian fluid flow through multiple stenotic arteries with non-uniform cross section. Advances and Applications in Fluid Mechanics, 21, 411-429 (2018). https://doi.org/10.17654/FM021040411 